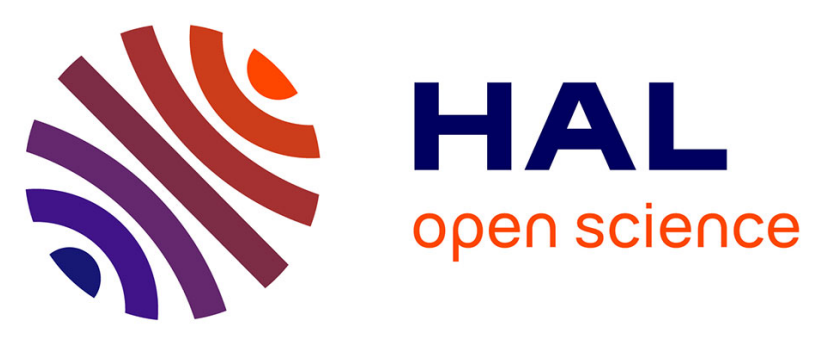

\title{
SME-4-producing Serratia marcescens from Argentina belonging to clade 2 of the S. marcescens phylogeny
}

Laura Dabos, Rafael Patino-Navarrete, Marcela Nastro, Angela Famiglietti, Philippe Glaser, Carlos Rodriguez, Thierry Naas

\section{- To cite this version:}

Laura Dabos, Rafael Patino-Navarrete, Marcela Nastro, Angela Famiglietti, Philippe Glaser, et al.. SME-4-producing Serratia marcescens from Argentina belonging to clade 2 of the S. marcescens phylogeny. Journal of Antimicrobial Chemotherapy, 2019, 74 (7), pp.1836-1841. 10.1093/jac/dkz115 . pasteur-02565488

\section{HAL Id: pasteur-02565488}

https://hal-pasteur.archives-ouvertes.fr/pasteur-02565488

Submitted on 6 May 2020

HAL is a multi-disciplinary open access archive for the deposit and dissemination of scientific research documents, whether they are published or not. The documents may come from teaching and research institutions in France or abroad, or from public or private research centers.
L'archive ouverte pluridisciplinaire HAL, est destinée au dépôt et à la diffusion de documents scientifiques de niveau recherche, publiés ou non, émanant des établissements d'enseignement et de recherche français ou étrangers, des laboratoires publics ou privés.

\section{(c) (1) $\$$}

Distributed under a Creative Commons Attribution - NonCommercial| 4.0 International 
2 SME-4 producing Serratia marcescens from Argentina belonging to the clade 2 of the $S$. 86. Fax: +33 1452163 40. E-mail: thierry.naas@aphp.fr Argentina.

Abstract: $261(\max 250)$

Word count Text: 2099

Figures: 2 marcescens phylogeny. Laura DABOS $^{1,2 \ddagger}$, Rafael PATIÑO-NAVARRETE ${ }^{2 \ddagger}$, Marcela NASTRO ${ }^{3}$, Angela $^{2}$ FAMIGLIETTI $^{3}$, Philippe GLASER ${ }^{2,4}$, Carlos H. RODRIGUEZ ${ }^{3}$, Thierry NAAS ${ }^{1,2,5,6 *}$ ${ }^{1}$ EA7361 "Structure, dynamic, function and expression of broad spectrum B-lactamases", Paris-Sud University, Faculty of Medicine, Le Kremlin-Bicêtre, France

${ }^{2}$ Joint research Unit EERA « Evolution and Ecology of Resistance to Antibiotics », Institut Pasteur-APHP-University Paris Sud, Paris, France

${ }^{3}$ Departamento de Bioquímica Clinica, Hospital de Clínicas José de San Martín, Facultad de Farmacia y Bioquímica, Universidad de Buenos Aires, Ciudad Autónoma de Buenos Aires, ${ }^{4}$ CNRS, UMR3525, Paris, France.

${ }^{5}$ Department of Bacteriology-Hygiene, Bicêtre Hospital, Assistance Publique - Hôpitaux de Paris, Le Kremlin-Bicêtre, France

${ }^{6}$ French National Reference Center for Antibiotic Resistance, Le Kremlin-Bicêtre, France ${ }^{\ddagger}$ Both authors contributed equally to this work.

Supplementary material: Figure S1 and S2; Table S1 and S2.

Running title: SME-4 producing S. marcescens from Argentina (45)

* Corresponding author: Thierry Naas, Service de Bactériologie-Hygiène, Hôpital de Bicêtre, 78 rue du Général Leclerc, 94275 Le Kremlin-Bicêtre Cedex, France. Phone: +33 1452129 


\section{Synopsis}

Background: SME serine carbapenemase are increasingly reported, especially from North and South America. Here, we describe an SME-4-producing Serratia marcescens (SME-Sm) clinical isolate from Argentina and compared its genome with other SME-Sm recovered from different countries.

Methods: S. marcescens isolates were characterized by WGS using Illumina technology, susceptibility testing and MIC determination. Carbapenemase activity was revealed by biochemical tests based on imipenem hydrolysis. Additionally, WGS of S. marcescens containing or not $b l a_{\mathrm{SME}}$ genes were retrieved from Genbank database. A whole-genome phylogenetic analysis based on non-recombinant core SNPs was inferred for $S$. marcescens complete genomes and for those encoding any bla $a_{\mathrm{SME}}$ variant.

Results: S. marcescens 163 was resistant to temocillin, aztreonam and carbapenems, remaining susceptible to expanded-spectrum cephalosporins. Analysis of WGS data of S. marcescens 163 revealed a genome of 5,139,329 bp, and a chromosomally-encoded class A $b_{\text {SME-4 }}$ carbapenemase gene, which was located on a genomic island closely related to SmarG1-1 of $S$. marcescens N11-02820. The comparison of the $S$. marcescens genomes revealed that all SME-Sm isolates possess this genomic island inserted at the same loci, form a well-defined subcluster of cluster I of $S$. marcescens clade 1, while S. marcescens 163 belonged to clade 2, suggesting that the SME-encoding genomic island may have been transferred between isolates from different clades.

Conclusions: To the best of our knowledge this is the first report of a SME-4-Sm from Argentina. Bla $a_{\mathrm{SME}-4}$ gene is located on a SmarG1-1-like genomic island. The genome of $S$. marcescens 163 belongs to clade 2, unlike all the other SME-Sm isolates that belong to a subcluster of clade I. 


\section{Introduction}

Currently, $\beta$-lactamase-mediated resistance does not spare even the newest and most powerful $\beta$-lactams (carbapenems), whose activity is challenged by carbapenemases. ${ }^{1}$ Class A carbapenemases may be chromosomally encoded (SME, NmcA, SFC-1, BIC-1, PenA, FPH-1, SHV-38), plasmid-encoded (FRI-1) or both (KPC, GES, IMI). ${ }^{2}$ Chromosomally-encoded class A carbapenemases have been identified in rare Gram-negative species that appeared sporadically in clinical or environmental samples since their first discovery, more than 20 years ago. ${ }^{2}$ SME-1 (for "Serratia marcescens enzyme") was first detected in England from two $S$. marcescens isolates collected in $1982 .{ }^{3,4}$ SME-1 has a broad hydrolysis spectrum that includes penicillins, cephalosporins, aztreonam, and carbapenems. ${ }^{1,4}$ The SME enzymes have been found exclusively in $S$. marcescens and the five variants differing by one or two amino acid substitutions have been found infrequently and sporadically in UK, across North and south America, in Switzerland and in Mexico., ${ }^{2,3-9}$ Recently, analysis of the genetic environment of bla $a_{\mathrm{SME}}$ genes in three Canadian $S$. marcescens isolates revealed that it is located on a novel cryptic prophage genomic island, SmarGI1-1. This genomic island can be excised and circularized, which probably contributes to its dissemination amongst $S$. marcescens..$^{10}$

In this work, we have characterized the genome of an SME-4-producing S. marcescens (SME-4-Sm) from a clinical isolate recovered from an University Hospital in Buenos Aires, Argentina, and compared it to the genomes of SME-Sm isolates either sequenced in this study or downloaded from the Genbank database.

\section{Materials and Methods}

Bacterial strains. S. marcescens 163 (Sm163) was identified with MALDI-TOF (MALDI Biotyper CA system, Bruker Daltonics, Billerica, MA, USA). Escherichia coli 
TOP10 (Invitrogen, Saint-Aubin, France) was used for cloning experiments. Bla $a_{\mathrm{SME}}$ gene harbouring Serratia marcescens, S. marcescens S6 (SmS6), ${ }^{3,4}$ S. marcescens S8 (SmS8), ${ }^{3,4} S$. marcescens CHE4 (SmCHE4), ${ }^{8} S$. marcescens AW (SmAW), ${ }^{5}$ S. marcescens USA-1 (SmUSA-1) and S. marcescens Mex-1 (SmMEX-1) were also used in this study. The presence of SME was systematically assessed on all the genome data of $S$. marcescens deposited on the NCBI genome database (December 2017). Genomes of 323 S. marcescens isolates were downloaded from Public databases including seven SME-Sm and 24 fully-sequenced S. marcescens strains (Tables S1 and S2). The sequences of the SmarGI1-1 and flanking regions from $S$. marcescens N10-0408, N11-2820 and N12-0620 were from accession numbers KF445086, KF318367 and KF615855, respectively, in the GenBank database. ${ }^{10}$

Antimicrobial agents, susceptibility testing. Antimicrobial susceptibilities and MIC values were determined as previously reported ${ }^{11}$ and interpreted according to the EUCAST breakpoints (16 May 2018; http://www.eucast.org/clinical_breakpoints/).

\section{Biochemical and molecular detection of carbapenemase-producing}

Enterobacteria. Carba NP test and $\beta$ Carba Test were used for carbapenemase detection (BioRad, Marnes-la-Coquette, France) as previously described. ${ }^{12,13}$ Genomic DNA of $S$. marcescens 163 isolate extracted using the Qiagen DNAamp kit (Qiagen) was used as template for amplification of the most prevalent carbapenemases $\left(b l a_{\mathrm{OXA}-48}, b l a_{\mathrm{KPC}}, b l a_{\mathrm{NDM}}\right.$, bla $_{\mathrm{VIM}}$, bla $\left._{\mathrm{IMP}}\right) .{ }^{14}$

Whole genome sequencing and bioinformatic analysis. The whole genome of Serratia marcescens 163 was sequenced using Illumina technology, as previously reported. ${ }^{11}$ The acquired antimicrobial resistance genes were identified by uploading assembled genomes to the Resfinder server v2.1 (http://cge.cbs.dtu.dk/services/ResFinder-2.1). ${ }^{15}$

ProgressiveMauve and the Mauve distributed script stripSubsetLCBs ${ }^{16}$ were used to extract all the regions with a minimun length of $500 \mathrm{bp}$ shared by all the strains, generating a 
102 2.877.168 bp core genome alignment. Recombinant variable positions were removed with

103 Gubbins (https://github.com/sanger-pathogens/gubbins), and a Maximum Likelihood

104 phylogeny was inferred for the alignment of the 631207 non-recombinant variable sites with

105 RAxML using a General Time Reversible (GTR) model incorporating a gamma distribution

106 rate among sites. ${ }^{17}$.In parallel a whole genome phylogeny was estimated for all the S.

107 marcescens retrieved from public databases with $\mathrm{kSNP}^{18}$ (Supplementary Material and

108 methods). All genomes were annotated with Prokka, ${ }^{19}$ and graphic representations were

109 generated with the genoplot ${ }^{20}$ library in $\mathrm{R}$.

110 Nucleotide sequence accession number. The whole genome sequences generated in

111 the study have been submitted to the Genbank nucleotide sequence database under the

112 accession number detailed in Table S1. The genomic islands constructed from whole genome

113 sequences available at Genbank nucleotide sequence database where submitted to the

114 Genbank Third Party Annotation database under accession numbers BK010577-BK010585

115 (Table S1). The nucleotide sequence of bla $a_{\mathrm{SRT}-3}$ gene has been submitted to the

116 EMBL/Genbank nucleotide sequence database under the accession number MG234451.

118 Results and discussion

119 Clinical case. A 62-year-old man with diffuse large B cell lymphoma was admitted at 120 the University hospital in Buenos Aires in July 2016 for his third cycle of chemotherapy.

121 During previous hospital stays in the same year he had received antimicrobial therapy with

122 meropenem, colistin and vancomycin. During his $3^{\text {rd }}$ stay the patient presented fevers and 123 developed pneumonia. He was treated with meropenem and oseltamivir, but cultures 124 remained negative. Twenty days after admission, a carbapenem-resistant S. marcescens 125 isolate was recovered from both a surveillance rectal swab and a catheter blood culture, 126 whereas the peripheral blood cultures remained negative. Further carbapenem-resistant $S$. 
127 marcescens isolates were recovered, from the same patient, in subsequent surveillance

128 samples for 3 weeks. The patient was discharged after having completed the $4^{\text {th }}$ cycle of

129 chemotherapy for his underlying disease and he did not suffer from subsequent infections.

$130 \quad$ Characteristics of $\boldsymbol{S}$. marcescens 163. S. marcescens 163 was resistant to amoxicillin

131 (MIC >256 mg/ml), temocillin (MIC of $24 \mathrm{mg} / \mathrm{L}$ ) aztreonam (MIC >256 mg/ml) and

132 carbapenems (MIC for imipenem, meropenem and ertapenem $>32 \mathrm{mg} / \mathrm{L}$ ), remaining

133 susceptible to expanded-spectrum cephalosporins (MIC for ceftazidime $1 \mathrm{mg} / \mathrm{L}$ ). $S$.

134 marcescens 163 also showed an MIC of $64 \mathrm{mg} / \mathrm{L}$ for amoxicillin+clavulanic acid. (Carba NP

135 and $\beta$ Carba tests gave positive results indicating the presence of a carbapenemase. PCRs

136 detecting the five most prevalent carbapenemases (bla OXA-48, $_{\text {B }}$ bla $\left.a_{\mathrm{KPC}}, b l a_{\mathrm{NDM}}, b l a_{\mathrm{VIM}}, b l a_{\mathrm{IMP}}\right)$

137 were negative. High level of resistance to amoxicillin and carbapenems, decrease MIC values

138 in presence of clavulanic acid and susceptibility to expanded-spectrum cephalosporins

139 suggested the presence of a bla $a_{\mathrm{SME}}$ gene.

140 WGS of $S$. marcescens 163 revealed a genome of 5,139,329 bp, with a 68 X coverage.

141 Resistome was determined by searching acquired genes and point mutations likely involved in

142 resistance. According to https://cge.cbs.dtu.dk/services/, the isolate presented two $\beta$-lactamase

143 genes, the chromosomal and natural $b l a_{\mathrm{STR}-3}$ gene coding for cephalosporinase and $b l a_{\mathrm{SME}-4}$

144 gene coding for a chromosomal class A carbapenemase (Table 1S). The isolate also harboured

145 the $S$. marcescens intrinsic aac(6')-Ic gene conferring resistance to amikacin and

146 gentamicin, ${ }^{21}$ and the tetracycline resistance gene tet(41). ${ }^{22}$ Isolates SmS6 and SmS8 also

147 harboured the streptomycin/spectinomycin resistance gene aadAl and the trimethoprim

148 resistant gene $d f r A l$.

149 WGS of $S$. marcescens 163 , revealed that the bla $a_{\mathrm{SME}-4}$ gene was inserted into a

150 SmarGI1-1 prophage-like genomic island initially described in three SME-1 producing $S$.

151 marcescens N11-02820, N10-00408 and N12-00620 isolates (accession numbers KF318367, 
$152 \mathrm{KF} 445086$, and KF615855, respectively). ${ }^{10}$ This genomic island found in Sm163 is $28.4 \mathrm{~kb}$

153 in size and bracketed by $25 \mathrm{bp}$ imperfect direct repeats (Figure 1A). While the upstream

154 sequences of the SmarGI1-1 were identical between Sm163 and N11-02820 isolates,

155 downstream of the attR site the sequences were similar except for an 8-kb deletion in $S$.

156 marcescens 163 that included genes coding for intCP $\Delta$, GNAT family, RES domain,

157 Helicase, ATP-dependent OLD family protein (Figure 1A).

158 Phylogeny of SME-producing S. marcescens. Phylogeny of the S. marcescens from

159 this study along with the 323 S. marcescens assemblies retrieved from the NCBI genome

160 database using KSNP3, revealed the two clades, with clade 1 representing $80 \%$ of the isolates

161 (Figure S1). Sixteen phylogenetic clusters were defined using ClusterPicker. 6 clusters were

162 located in Clade 1, with cluster 1 being the main one. In Clade 2, 10 clusters were identified,

163 with Sm163 beeing not related to any cluster. The presence of bla $a_{\mathrm{SME}}$ as well as other

164 carbapenemase genes was screened in the $323 \mathrm{~S}$. marcescens assemblies retrieved from the

165 NCBI genome database (Figure 1S). In total seven bla $a_{\mathrm{SME}}$ encoding isolates were found

166 (Table S1 and S2) that were added to the seven SME producers sequenced in the present

167 study. A core genome SNP-based Maximum Likelihood phylogenetic tree was obtained for

168 the fourteen SME-producing strains and 24 completed $S$. marcescens genomes retrieved from

169 the NCBI database (Table S1), using the S. marcescens fgi94 genome as outgroup. Two main

170 clades, with a mean genetic distance between them of 25.302 non-recombinant SNPs, were

171 revealed in the $S$. marcescens phylogeny (Figure 2, Table S1). The cluster numbers were

172 those from Figure S1 (Figure S1 and 2). Thirteen out of the fourteen SME-producers were

173 found on the clade 1, eleven of them formed a single sub-cluster of SME producers (hereafter

174 named SME sub-cluster within the cluster I), and the two remaining, MGH315 is located

175 outside the SME-sub-cluster but still in Cluster I, while BIDMC44 belongs to cluster III. On 
176 the other hand, S. marcescens 163, remains as the single strain encoding SME in clade 2 177 (Figure 2).

178 Eleven isolates encoded other carbapenemases than bla $a_{\mathrm{SME}}$ genes, 5 of them encoded $179 b l a_{\mathrm{KPC}}$ carbapenemase genes, 4 for $b l a_{\mathrm{NDM}-1}$ genes, one encoded bla $_{\mathrm{IMP}-1}$ gene and one $b l a_{\mathrm{VIM}-1}$ 180 gene (Figure S1, and Table S2). All of them were found in the clade 1, leaving $S m 163$ as the 181 only carbapenemase producing isolate from the clade 2.

182 Genetic environment of the bla $a_{\text {SME }}$ genes. In all cases the bla $a_{\mathrm{SME}}$ gene was encoded 183 within a conserved SmarGI1-1-like genomic island that varies in size from c.a. 27.2 (in $184 \mathrm{SmS6}$ ) to $28.5-\mathrm{Kb}$ (in BIDMC44) and that is integrated after the $s s r A$ gene (Figure $1 \mathrm{~A}$ and 185 1B, and Figure S2). ssrA has been identified as a hotspot for integration of mobile genetic 186 elements in enterobacteria. ${ }^{10}$ Three different flanking regions have been identified 187 downstream of the attR site among the 14 SME producers (Figure 1B). The first one 188 possesses a 2.8-kb long inserted region observed in the S. marcescens strains N12-00620 (and 189 N10-00408, data not shown $)^{10}$ that is shared by the strains that form the SME subcluster and 190 the strain MGH315 (Figure 1B and Figure S2). The second one possesses an 11.3-kb long 191 region as observed in the strain N11-02820. Finally, the third one has been described in the 192 Sm163 (Figure 1B), and in the strain BIDMC44, that is a member of clade 1 (Figure 2), 193 suggesting that the latter might be the donor of this structure.

194 The region downstream the ssrA gene was also analysed in the strains umh3 and 195 umh9, both of them are basal to the SME subcluster (Figure 2). In the strain umh3 an 196 incomplete SmarGI1-1 was identified (Figure 1C). It was structurally similar to the first 12.4

$197 \mathrm{~Kb}$ of the SmarGI1-1, but the hypothetical protein downstream the intA1 integrase gene was 198 substituted by three completely different genes coding for hypothetical proteins (Figure 1C).

199 No traces of the second half of the SmarGI1-1 could identified in the umh3 genome; however, 200 the attL and attR sites were present (Figure 1C). Not a single trace of SmarGI1-1 nor of the 
truncated version of it was found in the strain umh9 basal to both, umh3 and SME subcluster.

202 No traces for SmarGI1-1 were detected in the closest strains to the other SME-producers 203 analysed in the present study. Despite that the incomplete SmarGI1-1-like in strain umh3 204 shares a structural similarity with the first $12.4 \mathrm{~kb}$ of the SmarGI1-1, the sequence identity is 205 low (about 92\%), thus, ruling out the possibility that they share a recent ancestor. In fact, 206 there are a lot of recombination and exchanges between phages and other MGEs, which often 207 makes it impossible to build an evolutionary scenario. Interestingly, in the phylogeny performed with the 323 sequenced $S$. marcescens, a 209 single isolate GCF_000739215 lacking the SmarGI1-1 genomic island was found within the 210 the SME-subcluster (Figure 1S). In fact, in the isolate GCF_000739215, the insertion site 211 AttB was empty as identified in isolate umh9.

\section{Conclusion}

This is the first report of SME-4-Sm in Argentina. Until now, SME-4 had only been 215 reported in Brazil, ${ }^{7}$ and in one single SME-4 producing S. marcescens from US, as reported in 216 GenBank under the accession number KF481967, but not published yet. The increase in the 217 use of carbapenems in Argentina, ${ }^{23}$ together with the $S$. marcescens natural resistant to 218 polymyxin, could be factors that helped to select SME-producing $S$. marcescens isolates. 219 Finally, as increasing numbers of the SME carbapenemases are being reported from $S$. 220 marcescens, especially from North and South America, rapid detection systems based on 221 multiplex PCR analyses should also target bla $a_{\mathrm{SME}}$ genes in addition to the big 5 222 carbapenemases: KPC and OXA serine carbapenemases, and the IMP, VIM and NDM 223 metallo- $\beta$-lactamases, as was previously suggested by Bush et al. ${ }^{24}$ Thus, underdetection of 224 SME-producers may favor their silent spread. 
Finally, the phylogeny of all the available $S$. marcescens genomes assemblies revealed that all SME-Sm isolates possess a SmarGI1-1 inserted at the same loci, that they form a welldefined subcluster of cluster I of $S$. marcescens clade 1, while S. marcescens 163 belonged to clade 2, suggesting that the SmarGI1-1 may have been transferred between isolates from different clades.

\section{Acknowledgment}

233 We thank the Pasteur International Bioressources Network (Paris, France) for providing 234 whole genome sequencing facilities.

\section{Funding}

237 This work was supported by the Assistance Publique - Hôpitaux de Paris (AP-HP), the 238 University Paris-Sud, the Laboratory of Excellence in Research on Medication and Innovative 239 Therapeutics (LERMIT) supported by a grant from the French National Research Agency 240 [ANR-10-LABX-33] and by the Joint Programming Initiative on Antimicrobial Resistance 241 (JPIAMR) DesInMBL [ANR-14-JAMR-002], and by DIM Malinf, Ile de France, for LD's 242 PhD fellowship.

\section{Transparency declarations}

245 No competing interests to declare.

\section{References}

2481 Patel G, Bonomo R.A. Status report on carbapenemases: challenges and prospects. Expert 
2 Naas T, Dortet L, Iorga BI. Structural and Functional Aspects of Class A Carbapenemases. Current Drug Targets 2016; 17: 1006-28.

3 Naas T, Vandel L, Sougakoff W, et al. Cloning and sequence analysis of the gene for a carbapenem-hydrolyzing class A beta-lactamase, Sme-1, from Serratia marcescens $S 6$. Antimicrob Agents Chemother. 1994 38:1262-70.

4 Yang $\mathrm{Y}, \mathrm{Wu} \mathrm{P}$, Livermore $\mathrm{DM}$. Biochemical characterization of a $\beta$-lactamase that hydrolyzes penems and carbapenems from two Serratia marcescens isolates. Antimicrob. Agents Chemother. 1990; 34:755-8.

5 Poirel L, Wenger A, Bille J, et al. SME-2-producing Serratia marcescens isolate from Switzerland. Antimicrob Agents Chemother 2007; 51: 2282-3.

6 Queenan AM, Shang W, Schreckenberger P, et al. SME-3, a novel member of the Serratia marcescens SME family of carbapenem-hydrolyzing $\beta$-lactamases. Antimicrob Agents Chemother 2006; 50: 3485-7.

7 Cayô R, Leme RC, Streling AP, et al. Serratia marcescens harboring SME-4 in Brazil: A

8 Carrer A, Poirel L, Pitout JD, et al. Occurrence of an SME-2-producing Serratia marcescens isolate in Canada. Int J Antimicrob Agents 2008; 31: 181-2.

9 Hopkins KL, Findlay J, Meunier

$\mathrm{D}$, et al. Serratia marcescens producing SME carbapenemases: an emerging resistance problem in the UK? J Antimicrob Chemother. 2017; 72:1535-7.

10 Mataseje LF, Boyd DA, Delport J, et al. Serratia marcescens harbouring SME-type class A carbapenemases in Canada and the presence of blaSME on a novel genomic island, SmarGI1 1. J Antimicrob Chemother 2014; 69: 1825-9. 
27311 Dabos L, Bogaerts P, Bonnin RA, et al. Genetic and biochemical characterization of OXA274 519, a novel OXA-48-like $\beta$-lactamase. Antimicrob Agents Chemother 2018; $275 \quad 62: \mathrm{e} 00469-18$.

27612 Nordmann P, Poirel L, Dortet L. Rapid detection of carbapenemase-producing 277 Enterobacteriacea. Emerg Infect Dis 2012; 18:1503-7.

27813 Bernabeu S, Dortet L, Naas T. Evaluation of the $\beta$-CARBA ${ }^{\mathrm{TM}}$ test, a colorimetric test for 279 the rapid detection of carbapenemase activity in Gram-negative bacilli. J Antimicrob $280 \quad$ Chemother 2017; 72: 1646-58.

14 Samuelsen O, Overballe-Petersen S,Bjørnholt JV, et al. Molecular and epidemiological 282 characterization of carbapenemase-producing Enterobacteriaceae in Norway, 2007 to 2014. PLoS One 2017; 12: e 0187832.

15 Zankari E, Hasman $\mathrm{H}$, Cosentino $\mathrm{S}$, et al. Identification of acquired antimicrobial 285 resistance genes. J Antimicrob Chemother 2012; 67: 2640-4.

16 Darling AE, Mau B, Perna NT. Progressive Mauve: multiple genome alignment with gene gain, loss and rearrangement. PLoS One 2010; 5: e11147.

17 Stamatakis A. RAxML-VI-HPC: maximum likelihood-based phylogenetic analyses with thousands of taxa and mixed models. Bioinformatics 2006; 22: 2688-90.

18. Gardner, S.N. and Hall, B.G. When whole-genome alignments just won't work: kSNP v2 software for alignment-free SNP discovery and phylogenetics of hundreds of microbial genomes. PLoS ONE 2013; 8: e81760.

19 Seemann T. Prokka: rapid prokaryotic genome annotation. Bioinformatics 2014; 30: 20689.

20 Guy L, Kultima JR, Andersson SG. genoPlotR: comparative gene and genome 296 visualization in R. Bioinformatics 2010; 26: 2334-5. 
29721 Shaw KJ, Rather PN, Sabatelli FJ, et al. Characterization of the chromosomal aac(6')-Ic gene from Serratia marcescens. Antimicrob Agents Chemother 1992; 36: 1447-55.

22 Thompson SA, Maani EV, Lindell AH, et al. Novel tetracycline resistance determinant isolated from an environmental strain of Serratia marcescens. Appl Environ Microbiol 2007; 73:2199-206

23 Quiroga C, Nastro M, Di Conza, J. Current scenario of plasmid-mediated colistin resistance in Latin America. Rev Argent Microbiol 2018; pii: S0325-7541(18)30052-X

24 Bush K, Pannell M, Lock JL et al. Detection systems for carbapenemase gene identification should include the SME serine carbapenemase. Int J Antimicrob Agents $2013 ; 41: 1-4$.

30725 Mao C, Bhardwaj K, Sharkady SM, et al. Variations on the tmRNA gene. RNA Biol $308 \quad 2009 ; 6: 355-61$.

309 


\section{Figure legends}

Figure 1. (A). Schematic representation of the genetic structure of SmarGI1-1 of $S$. marcescens N11-02820 (KF318367) and S. marcescens 163 and flanking regions. Common structures are highlighted in gray. Genes and putative functions of genes are shown; $\mathrm{H}$ means hypothetical protein. (B). Comparison of the SmarGI1-1 and its genetic environments among the different bla $a_{\mathrm{SME}}$-encoding strains. (C). Comparison of the insertion site for SmarGI1-1 in the SME subcluster and its basal strains. Numbers below the representative SME cluster represent: 1 . Phage regulatory protein, 2. Phage capside protein, 3. AlpA phage transcriptional regulator, CI repressor-like protein, 5. primase. $\mathrm{H}$ above the CDS B) AttL and AttR sequences comparison between umh3 and SmarGI1-1 (UCI-88).

Arrows colored in blue, green, purple, red and yellow correspond to genomic CDS, variable genomic CDS, TmRNA ${ }^{25}$, Bla $a_{\mathrm{SME}}$ gene, and genomic island CDS, respectively. Horizontal bars (I) in black and in red represents, Att (L and R) sites and contig boundaries, respectively. Small light green boses ( ) represent RNA genes. Insertion sequences are indicated by an arrow in a rectangle light green for ISEc31 and ISSm3, and grey for the interrupted ISEc14.

Figure 2. S. marcescens Maximum Likelihood tree obtained from core non-recombinant SNPs. All bla $a_{\mathrm{SME}}$-encoding strains were bolded and capital letters. Those sequences in this study are highlighted in grey, and bla $a_{\mathrm{SME}-2}$-expressing isolates are underlined. $S$. marcescens isolates not carrying $b l a_{\mathrm{SME}}$ gene are represented in lower case letters. 
(A)

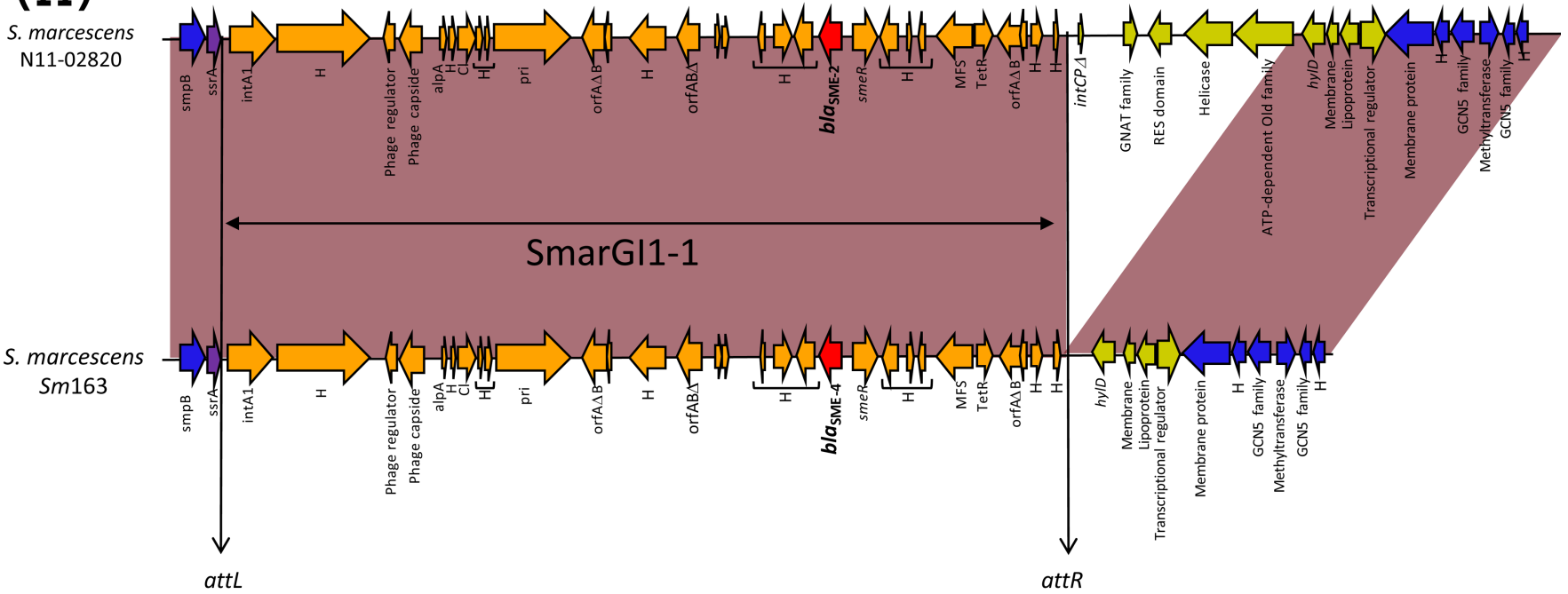

cgggggttcaaatcccccagctccaccaaatgatgatccgg N11-02820 taatacataaaftcatattcccccagctccaccaaa-a-ttctccat

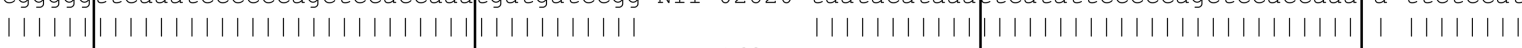
cgggggttcaaatcccccagctccaccaaatgatgatccgg sm163 taatacataaatcatattcccccagctccaccaaa 3 acttctccat

(B)

$\mathrm{N} 12-00620$ attL $\rightarrow$ ISEC31 ISSM3 bla

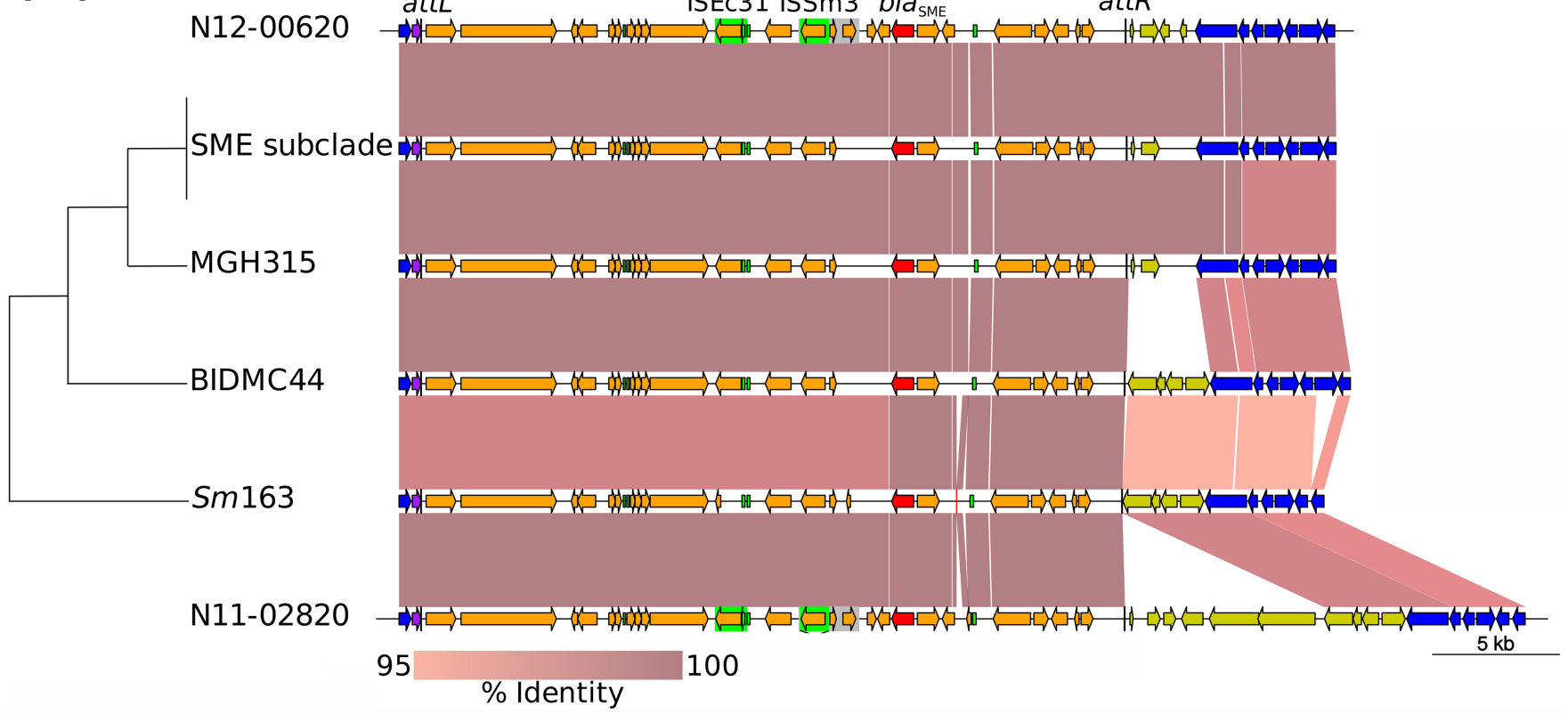

\section{(C)}

attL $\quad H H$

1) $\mathrm{N} 12-00620$ 制二 $\longrightarrow$
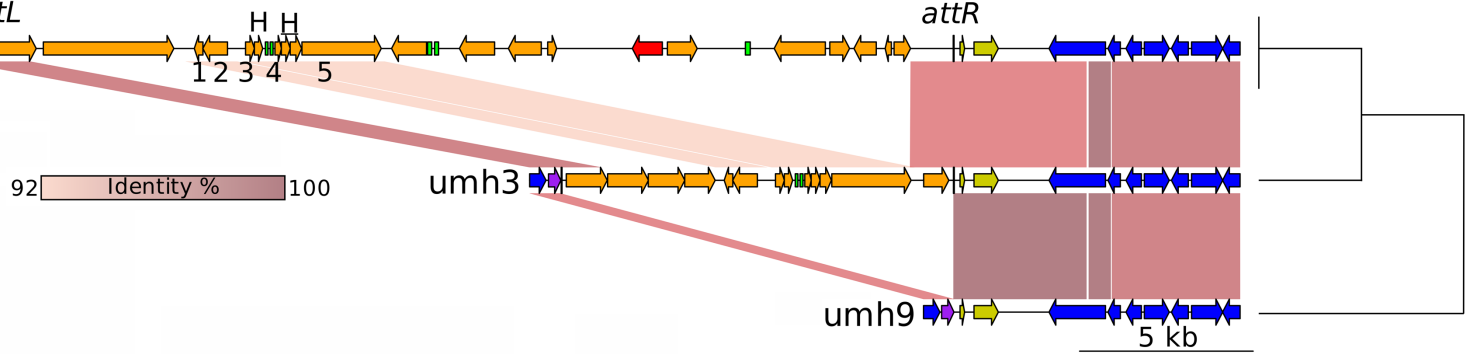

2 ) tgccgacggtgagtaatttcggacgcgggttcaactcccgccagctccaccacat umh3 gttaataaatacattcagattcccccagctccaccaaatacgtcgccagtgaacca

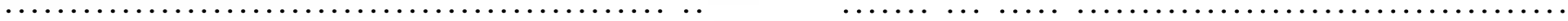
tgccgacggtgagtaatttcggacgcgggttcaactcccgccagctccaccaaat N12-00620 gttaatacataaattcatattcccccagctccaccaaatacgtcgccagtgaacca attl

3 ) aatttcgggacacađattcaactcccaccađctccaccaaatacatcgccagtgaa

$\underset{3,193,266}{\downarrow} \begin{array}{ccc}\text { attB } & \perp \\ \text { umh9 } & \text { 3,193,290 }\end{array}$

Fig. 1 


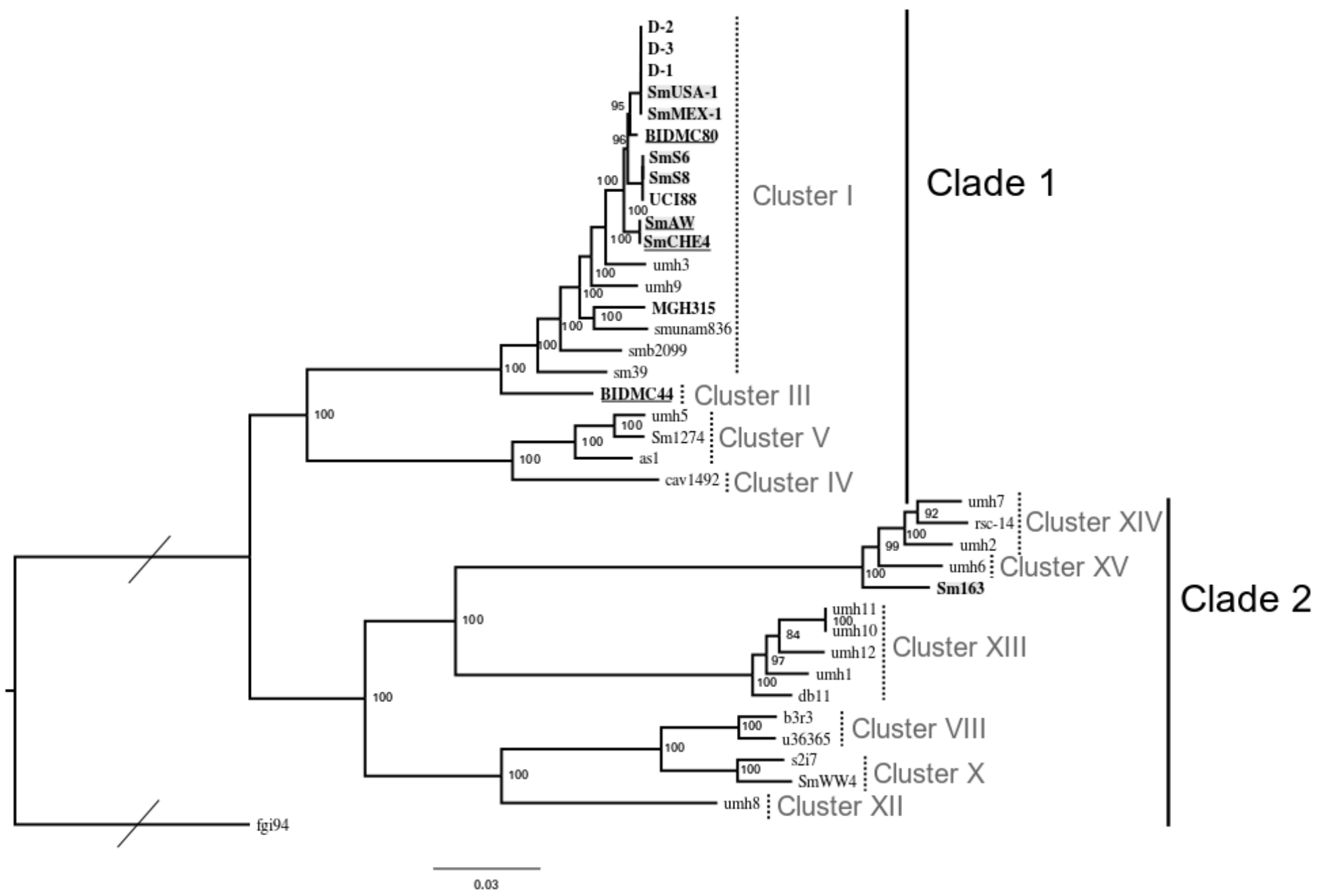

Fig. 2 